Research Article

\title{
Tissue Engineering Scaffold Materials to Repair Sports Cartilage Injury
}

\author{
Mo Xing (i) \\ School of Physical Education, Hulunbuir University, Hulunbuir 021000, Inner Mongolia, China \\ Correspondence should be addressed to Mo Xing; xingmo@hlbec.edu.cn
}

Received 19 May 2021; Revised 7 June 2021; Accepted 14 June 2021; Published 2 July 2021

Academic Editor: Sang-Bing Tsai

Copyright (C) 2021 Mo Xing. This is an open access article distributed under the Creative Commons Attribution License, which permits unrestricted use, distribution, and reproduction in any medium, provided the original work is properly cited.

\begin{abstract}
With the continued development of sports in China, sports sometimes cause cartilage damage. The purpose of this research is to study the tissue engineering scaffold material for sports cartilage damage repair. In this study, mesenchymal rat bone marrow stem cells (to put it simply, stem cells are a type of cell with unlimited or immortal self-renewal capacity, capable of producing at least one type of highly differentiated progeny cells) were obtained by the total bone wash method. The cells were inoculated into the cell culture bottle. When the primary cultured cells proliferated to about $80 \%$ of the culture bottle area, the cells were digested with trypsin to open the cell link, then the medium containing $10 \%$ serum was added to terminate the cell digestion, and then the passage expansion was carried out according to the cell density. PLGA/NHA and PLGA were heated to $65^{\circ} \mathrm{C}$ under ultrasonic vibration until uniform PLGA/NHA and PLGA solutions were obtained. Then, the samples were added to the tube mold and then heated and cooled to obtain the composite porous scaffold of mesenchymal stem cells. $10 \mu \mathrm{l}$ MSCs cell suspension was extracted with a microinjector, and the needle was injected from the inside of the scaffold, and the cell suspension was added outside the scaffold to ensure that there were composite cells inside and outside the scaffold. The subcutaneous tissue of the skin was cut along the medial side of the knee joint and the capsule of the switch segment was cut. The scaffold materials were filled into the osteochondral defect to observe the cartilage healing. The mechanical strength of $0.5 \%$ PLGA-MSCs composite porous scaffold was increased to $1.1 \mathrm{MPa}$, and the cell density was high. The repair of cartilage in rats was the best. The results showed that the porous scaffolds designed in this study have good compatibility and are beneficial to repair sports cartilage injury.
\end{abstract}

\section{Introduction}

The survival of engineered tissue constructs in the initial stage after implantation depends on the rapid development of proper blood vessel formation. On the contrary, this is the main condition for maintaining the durability of this structure. "Prevascularization" is a medical technology that is expected to be a major breakthrough in tissue engineering, which aims to generate preformed microvasculature in tissue constructs before implantation in tissue. Situ method treats the human body as a natural bioreactor and causes vascularization and arteriovenous circulation through angiogenesis, growth, or skin. In the future, these strategies can be complemented with microvascular transplantation of adipose tissue fragments or producing extremely organized microvascular networks in vitro by advanced microtechnology and microreflection systems. The further development of these prevascular concepts and their adaptability to individual therapeutic interventions will significantly promote the widespread implementation of tissue engineering applications in clinical practice.

Human soft tissue injury is a common and frequently occurring disease in the human locomotor system. Many people suffer from this disease at home and abroad. Soft tissue injuries can cause different conditions and symptoms in neck, shoulder, back, waist, legs, and limbs due to acute injury and chronic cumulative damage, and some even induce many different symptoms similar to internal medicine, neurology, and gynecology. However, for such diseases that are directly caused by soft tissue damage, in the past, traditionally, they tended to find the cause in the skeletal system, or even if they knew it, there was a lack of in-depth 
systematic research. In recent decades, through continuous observation and research by colleagues in the medical field, it has been found that a large part of many diseases are directly caused by soft tissue muscles, ligaments, fascia, fat, joint capsules, nerves, and blood vessels, which is harmful. Therefore, domestic and foreign scholars have made a comprehensive and systematic exploration and research work on the disease. In recent years, hospitals in various places have begun to establish specialties for the treatment of soft tissue injuries, which has made the subject of soft tissue injury recognized and accepted by more and more colleagues in the medical field. Soft tissue engineering is a newly developed strategy to repair damaged soft tissues and organs, and it broadens new ideas based on existing methods. Collagen fibers support most of the cartilage tissue of the human body and thus form a three-dimensional microstructure. The fiber-reinforced scaffold mimics the structural steel advantages of natural soft tissue machinery and biological environment and provides the possibility for soft tissue regeneration and remodeling. The latest research progress stent design and production of this study to enhance and add fiber blessing in vitro and in vivo structural features of the new fiber used for soft tissue repair and mechanical strength and influence biological activity were analyzed. The microstructure of the fiber-reinforced scaffold can be adjusted, and its mechanical properties and degradation rate can provide a satisfactory development platform for biological functional implants in soft tissue engineering or regenerative medicine.

The ultimate goal of tissue engineering is to repair and restore tissues or organs damaged by accidents or diseases. Yong et al. aim to study the feasibility of processing circulating polylactic acid (PDLLA)/hydroxyapatite (HA) biomaterials into tissue engineering scaffolds (TES) with variable mechanical properties and good interconnections. They applied the internally constructed bone scaffold 3D printing (BS 3P) system to two biological materials, namely, PDLLA-PCL and HA-PCL. Their research lacks experimental data [1]. Masoudi et al. believe that blood platelets derivatives are widely used in various fields of medicine and stem cell-based tissue engineering. They reviewed the range of platelet-rich blood products, discussed their current applications in tissue engineering and regenerative medicine, which reflected their effects on stem cells, and emphasized the current translation challenges. Their research process is very complicated [2]. Naumenko et al. used freezedrying to produce a porous biopolymer hydrogel containing 3-6 wt $\%$ natural clay nanotubes with $50 \mathrm{~nm}$ diameter $/ 0.8 \mu \mathrm{m}$ length, without any cross-linking agent. They used an enhanced darkfield microscope to observe the distribution of halloysite nanotubes at the implantation site. Their research lacks comparative experiments [3]. Emmert et al. believe that the control of cells with signs of life is seriously related to the survival of organisms. They studied chemistry as an external mechanical function of cells, especially cell migration control and analyzed this effect of control of the intracellular transport of information. Their research mainly includes quantitative analysis of the following aspects: (1) understanding the response changes of the intracellular state to external clues through the transportation of intracellular vesicles; (2) the perception of cells to external chemotactic cues; and (3) cell migration in 3D ability. Their research is not practical [4]. Tangsadthakun et al. believe that the biopolymer mixture between collagen and chitosan has the potential to produce biocompatible cell scaffolds. The porous scaffold is made by freeze-drying collagen and chitosan solution and cross-linking through dehydration heat treatment (DHT). They made various types of scaffolds by changing the composition of collagen and chitosan. The stent was fully characterized by Fourier Transform Infrared (FT-IR) spectroscopy. Their research process lacks data [5].

In this study, rat bone marrow mesenchymal stem cells were obtained by the method of washing a whole marrow. The cells were inoculated into cell culture flasks. When the primary cultured cells proliferated to about $80 \%$ of the culture flask area, trypsin digestion was used to open the cell link. Then $10 \%$ serum-containing medium was added to stop the cell digestion, and subculture expansion was carried out based on cell density. PLGA/NHA and polylactic acid-glycolic acid (PLGA) were heated to $65^{\circ} \mathrm{C}$ under ultrasonic vibration until a uniform PLGA/NHA and PLGA solution was obtained. Then, the sample was added to the tubular mold, heated, and cooled to obtain the mesenchymal stem cell composite porous scaffold. A microinjector was used to draw $10 \mu \mathrm{l}$ of MSCs cell suspension into the needle from the inside of the stent, and the cell suspension was dropped on the outside of the stent to ensure that there were composite cells inside and outside the stent. The skin subcutaneous tissue along the inner side of the rat's knee joint and the nodular sac were cut. The stent materials were filled into the osteochondral defect to observe the cartilage healing of the rat. The experimental object used in this article is rats, and the experimental results have research value, but they are not clinically feasible.

\section{Sports Cartilage Injury}

2.1. Tissue Engineering. In recent years, tissue engineering (TE) and regenerative medicine (RM) are growing field of potential applications of research and ideas. This is why regeneration and repair of human tissue and organ function lost the desire and longing $[6,7]$. TE combines the cells and extracellular matrix components of the putative stem cell characteristics with growth factors and support matrix to achieve independent tissue growth. In recent years, many scholars have gained insights on the interaction between cells and the manipulation of cells. However, so far, no ideal cell source has been found. In addition to the well-known sources of stem cells, because of their potential effects on TE and RM, cells (TC) have also attracted more and more attention in recent years [8-10]. It is becoming more and more obvious that TC provides regenerative potential and plays a role in cellular communication through the terminal poles that form the network. In recent decades, the manufacture of additives (also known as 3D printing) has become a disturbing technology for rapid prototyping and manufacturing [11]. Curing roller, bed melt powder, extruder, and injection molding binder are unique additive 
manufacturing technologies that have been widely used in many fields, including biomedical research and tissue engineering. With controlled biocompatibility of macropore and micropore printing, patient-specific geometry, and the ability to incorporate cells, drugs, and proteins, 3D printing is an ideal choice for orthopedic applications such as bone grafting $[12,13]$.

2.2. Application of Tissue Engineering. Cross-linked HA-Ge hydrogel has important viscosity and biological properties for vocal cord tissue engineering. Patient-specific viscosity adaptations of injectable biomaterials may improve tissue regeneration. The frequency-dependent viscoelasticity of the cross-linked HA-Kudzu hydrogel is measured as a function of the concentration of HA, Ge, and cross-linker [14]. The synthetic extracellular hydrogel matrix consists of HA and Ge modified with thiol and cross-linked polyethylene glycerin $[9,15]$. A newly developed characterization method based on Rayleigh wave propagation has been used to quantify the viscosity properties depending on the frequency of these hydrogels, including energy shear and loss factor over a wide frequency range [16]. This is $40-4000 \mathrm{~Hz}$. The viscosity properties of hydrogels increase with frequency. The value of the energy storage and loss modulus and the rate of frequency increase vary with the concentration of the component. Generally, the equilibrium contact angle $\theta$ (it refers to the angle between the solid-liquid interface and the gas-liquid interface from the solid-liquid interface to the gasliquid interface at the junction of solid, liquid, and gas) $\theta$ and the three-phase interfacial tension should satisfy the following relationship:

$$
\lambda-\beta=\mu \cos \theta
$$

Among them, $\lambda, \mu$, and $\beta$ are the interfacial tension between solid-gas, gas-liquid, and liquid-solid $[17,18]$.

$$
\begin{aligned}
& A_{1}=\lambda+\mu-\beta=\mu(1+\cos \theta), \\
& A_{2}=\lambda-\beta=\mu \cos \theta, \\
& W_{s}=\lambda-\mu-\beta=\mu(\cos \theta-1) .
\end{aligned}
$$

Among them, $A_{1}, A_{2}$, and $W_{s}$ are adhesion work, wetting work, and spreading coefficient, respectively [19].

Over the centuries, breakthroughs have been made in the technical field of skin regeneration, and good progress has been made in wound healing, which has allowed the skin to recover faster. The skin mainly uses a scaffold matrix that matches the skin for regeneration. There are many types of scaffold matrix, and different scaffolds have different advantages and disadvantages. These scaffolds are composed of natural biomaterials or synthetic materials with very high biocompatibility. Scaffolds are made from natural biomaterials and synthetic biomaterials composition, and they have a very high biocompatibility, which can increase the tensile strength of tissue regeneration of the skin with a good performance. With the advancement of stem cell research and the development of smart biomaterials and three- dimensional biomanufacturing strategies, highly imitated tissues or organs can be designed. Among all biomanufacturing methods, bioprinting based on ink-jet printing technology is expected to deliver and manufacture bioimitation tissues with high throughput, digital control, and single-cell operation capabilities. Therefore, this technology has great potential in regenerative medicine and transitional applications. Further, side effects of benign bioprinted mammalian cells for gene may be printed or drug delivery; tissue construct can be used for the precise placement of cells conveniently achieved in the process $[20,21]$. By layer-by-layer assembly, the converted medical images can be used to print three-dimensional tissues with complex structures [22].

The discovery of interesting intrinsic properties of two-dimensional grapheme Na-mi materials has advanced further research and development in various electronics applications from biomedicine. Before long, several grapheme and grapheme-derived materials, because of their good conductivity, are widely used as components of diagnostics or biosensors. Graphene-based Na-mi materials stem cells can promote the adhesion, growth, expansion, and differentiation. Although the cell survival rate in vitro is not affected, its potential Na-mi materials can still play a role in the clinical environment. Therefore, strict international standardized clinical research is required on the human body to evaluate its $\mathrm{Na}$-mi materials $[23,24]$.

2.3. Cartilage Damage. Cartilage damage is the most common disease and the main reason of bruising pain. The current treatment for bruising pain can give patients a period of relief; there is still a great need for alternative tissue engineering solutions. In particular, it is necessary to develop a method that can completely solve the cartilage damage and restore the patient's body. The absolute premise of tissue engineering is to understand normal structure and function. Bone transplantation is currently one of the most common surgical procedures worldwide, but due to the high risk of complications and the lack of viable donor tissue, it is necessary to develop methods to replace bone defects. Combining the biocompatibility of stent and mesenchymal stem cells, tissue engineering could become a solution to the problem, but for the implementation of this program, there are still many obstacles and challenges. Dobby loom weft jet design and placement system and a warp tension control system automatically collect compatible bobbin, overcome the physical limitations caused by the inherent structure of the polymer, and minimize the polymer fiber damage $[25,26]$.

\section{Sports Cartilage Injury Repair Experiment}

3.1. Experimental Animals. SD rats, male, 12 weeks to 14 weeks, body weight $100 \mathrm{~g}$, were used to obtain bone marrow stem cells. The main reagents of the experiment are shown in Table 1.

3.2. Isolation and Culture of MSCs. SPSS is the general term for a series of software products and related services for statistical analysis operations, data mining, predictive 
TABLE 1: The main reagents of the experiment.

\begin{tabular}{|c|c|c|}
\hline Numbers & Reagent & Factory \\
\hline 1 & a-MEM medium & Gibco company \\
\hline 2 & $\begin{array}{c}\text { Australian newborn fetal bovine } \\
\text { serum }\end{array}$ & Gibco company \\
\hline 3 & CM-DIL & Molecular Probes \\
\hline 4 & Ficoll-Paque separation liquid & Pharmacia company \\
\hline 5 & EDTA & Gibco company \\
\hline
\end{tabular}

CM-DIL is a live cell stain.

analysis, and decision support tasks launched by IBM. There are Windows and Mac OS X versions. In this study, the rat bone marrow mesenchymal stem cells were obtained by the method of whole bone marrow washing. Three SD rats weighing about $100 \mathrm{~g}$ were severed and sacrificed and soaked in alcohol for about 10 minutes, and the rat femurs were isolated under aseptic conditions and removed with scissors. The muscles, tendons, and other tissues attached to the femur are cut off with scissors at both ends of the bone rice, and the femoral bone marrow cavity is repeatedly washed with PBS, washed with an equal amount of PBS without calcium and magnesium, and centrifuged for 10 minutes with a $900 \mathrm{~g}$ horizontal centrifuge. The fat layer and supernatant were discarded. The pellet was resuspended with a-MEM culture medium containing 10\% FBS and slowly added to the separation medium with a density of $1.077 \mathrm{~g} / \mathrm{ml}$ and centrifuged for 20 minutes in a $900 \mathrm{~g}$ horizontal centrifuge, and finally the mononuclear cell layer was collected and washed with PBS. After 3 times, the cells were resuspended in a-MEM medium containing $10 \%$ FBS and counted. The cells were inoculated in a cell culture flask, placed in an incubator at $37^{\circ} \mathrm{C}$ and $5 \% \mathrm{CO} 2$ concentration, and then cultured in an incubator with a concentration of 5\% CO2. After 48 hours, the medium was changed semiquantitatively and then every 3-4 days. When the primary cultured cells proliferate to about $80 \%$ of the culture flask area, trypsinization is used to open the cell link, and the cells are continuously piped to flow down, and then $10 \%$ serum-containing medium is added to terminate the cell digestion. The cell density is passaged and expanded.

\subsection{Construction and Performance Measurement of Mesenchymal Stem Cell Composite Porous Scaffold}

3.3.1. Construction of Composite Porous Scaffold of Mesenchymal Stem Cells. The nano-NHA powder was added to the 1,4-dioxane/water mixture and mixed with ultrasonic vibration. PLGA (LA/GA = 72:28) was added to that solution. The ratio of nanohydroxyapatite (NHA) powder/PLGA is 1 : 9. PLGA/NHA and polylactic acid-glycolic acid (PLGA) were heated to $65^{\circ} \mathrm{C}$ under ultrasonic vibration until a uniform PLGA/NHA and PLGA solution was obtained. Then, the sample was added to the tube mold and heated to $15^{\circ} \mathrm{C}$ higher than the cloud point temperature and then quickly placed in a constant temperature of $9^{\circ} \mathrm{C}$. After 1 hour, the mold was removed from the quenching tank, with rapid immersion in liquid nitrogen and the samples after annealing. Finally, the samples were frozen at $-60^{\circ} \mathrm{C} 14 \mathrm{kPa}$ and dried for 4 hours to remove the solution and to obtain a porous scaffold. The obtained porous scaffold composite was cut into $5 \mathrm{~mm}$ and $3 \mathrm{~mm}$ cylinders and sterilized with ethylene oxide.

\subsubsection{Determination of the Water Absorption Rate of the} Stent. The columnar stents with PLGA/NHA content of $0 \%$, $0.1 \%, 0.3 \%$, and $0.5 \%$ were prepared. Then about $0.2 \mathrm{~g}$ of the prepared scaffold was taken and put into a $4 \mathrm{~mL}$ centrifuge tube to weigh it, then put in a $50^{\circ} \mathrm{C}$ oven, and dried for $2-3$ days. After taking it out, the mass was weighted and the water absorption was calculated.

3.3.3. Mechanical Properties of the Bracket. The columnar stents with PLGA/NHA content of $0 \%, 0.1 \%, 0.3 \%$, and $0.5 \%$ were prepared, and the mechanical properties under compression conditions were measured with a universal tensile testing machine.

3.4. Compound of Cells and Scaffold. The stent material was irradiated with cobalt 60 for 10 minutes and sterilized for use. Using a microinjector, $10 \mu \mathrm{l}$ of MSCs cell suspension was drawn each time, the needle is inserted from the center of the longitudinal axis of the PLGA/NHA stent, then slowly withdrawn while injecting the cell suspension, and injection is done multiple times until the cell suspension overflows, and the cells are directly dripped on the surface of the stent suspension to ensure that there are composite cells inside and outside the stent.

3.5. Establishment of an Animal Model Cartilage Defect. $0.4 \%$ sodium pentobarbital $(2 \mathrm{ml} / \mathrm{kg}$ body weight $)$ was injected intraperitoneally for anesthesia. After successful injection, the SD rats of 12-13 weeks were supine on the operating table with their limbs fixed. After skin preparation, routine disinfection, and sterile hole towels, the skin subcutaneous tissue is cut along the inside of the rat's knee joint and the nodular capsule is cut, the patella is pushed and tailed outward, the knee joint femur trochlea is lined, and a dental drill is used to make a cylindrical osteochondral defect with a diameter of $1 \mathrm{~mm}$; the depth is about $2 \mathrm{~mm}$.

Experimental groups: blank group, MSCs-PLGA group (polylactic acid-glycolic acid copolymer is formed by random polymerization of two monomers, lactic acid and glycolic acid; it is a degradable functional polymer organic compound), and MSCs-PLGANHA group. The abovementioned grouping materials are filled into the osteochondral defect, respectively. After confirming that the stent completely fills the defect, the joint capsule is sutured and the skin incision is sutured and then simply bandaged. The knee joints were not immobilized. The mice were put in a cage and clean food and water were provided for them, and the room temperature was kept constant. Penicillin was injected intramuscularly for 3 days after the operation. 
3.6. Animal Observation. The rats' incision healing, active gait, and signs of infection are observed every day after surgery. At 6 weeks and 12 weeks after operation, the rats were sacrificed by neck dislocation, and the articular surface including the lower end of the femur was taken. Gross specimen observation: indicators such as the integration, flatness, and color of the regenerated cartilage between the regenerated tissue and the surrounding tissues are observed. Microscopic histological observation: the specimen is taken, fixed with $10 \%$ formalin for 12 hours, and decalcified with EDTA decalcification solution for 15 days. The bone and cartilage tissue containing the regeneration area along the vertical articular surface was cut, and HE staining and toluidine blue staining were performed.

3.7. Experimental Study on the Survival of Transplanted MSCs in Rat Cartilage Defects. The other 4 cartilage defects were implanted with CM-DIL labeled ADSCs for in vivo cartilage defect repair and MSCs survival experiments in rat cartilage defects. After 6 weeks and 12 weeks, the specimens were collected, fixed in paraformaldehyde solution for 3 days, decalcified with EDTA solution for 20 days, directly frozen, in sections of $12 \mu \mathrm{m}$ thickness, and observed with a laser confocal microscope.

\section{Sports Cartilage Injury Repair}

4.1. Comparison of Histological Scoring Results. A modified histological scoring standard was used to semiquantitatively evaluate the repaired tissue at 12 weeks. SPSS is the general term for a series of software products and related services for statistical analysis operations, data mining, predictive analysis, and decision support tasks launched by IBM. There are Windows and Mac OS X versions. The comparison of histological scores is shown in Table 2. The average matrix score, the thickness of new cartilage, and uterine type II collagen staining in the PLGA/NHA group are better than the PLGA group and the blind control group, which indicates that the hyaline cartilage regeneration rate in the PLGA/NHA group is significantly higher than that of the PLGA group and the control group. The average subchondral bone score of the PLGA/NHA group was higher than that of the PLGA group and the blank control group. Therefore, the subcontract bone formation in the PLGA/ NHA group was more than that in the PLGA group and the control group. The total score of the PLGA/NHA group is higher than that of the PLGA group and the control group, indicating that the osteochondral repair of the PLGA/NHA group is better than that of the PLGA group and the control group. Morphology scanning electron microscope (SEM) showed that TES formed from these materials may exhibit two new cellular structures, having excellent fusion, high uniformity, and TES characteristics of fully interconnected control channel. Typical compression deformation behavior of porous materials meets the test. In vitro cell culture studies and confocal laser microscopy (CLM) display cell adhesion, proliferation, and extracellular matrix (ECM) formation increase. The results demonstrate novel biocompatible biomaterial scaffolds can enhance cell. Cell viability refers to the percentage of live cells among the total cells that die due to various reasons in the cell population.

4.2. Cell Growth and Proliferation in the Scaffold. Stent cell growth and proliferation are shown in Figure 1. The results showed that the cell activity of the polylactic acid-glycolic acid and mesenchymal stem cell composite porous scaffold group on the 9th day increased 8 times and 9 times, respectively. This indicates that the cells increased rapidly in the first 9 days. However, the cell viability of the polylactic acid-glycolic acid and mesenchymal stem cell composite porous scaffold group increased only 1.16 times and 1.17 times on day 17 compared with that on day 9. This indicates that the cell proliferation slows down during this period. On the 17th day, the cell viability of the PLGA/NHA scaffold group was significantly higher than that of the PLGA group $(p<0.05)$. Fluorescence quantitative DNA method has high sensitivity and strong specificity, and it is a reliable method for detecting cell proliferation. When Hochest33258 binds to double-stranded DNA, it can produce fluorescence, and the intensity of this fluorescence is directly proportional to the number of cells. The number of cells is calculated by the intensity of fluorescence. Our results showed that the cell DNA content of PLGA and PLGA/NHA scaffold group on day 9 increased 13.3 times and 14.1 times, respectively, compared with day 0 . However, the cell viability of the PLGA and PLGA/NHA scaffold group on the 17th day only increased 1.4 times compared with the 9th day. This indicates that the DNA quantitative analysis result is equal to the MTT result. The DNA content of the PLGA stent group was significantly lower than the PLGANHA group on the 9th and 17th day, which indicates that the PLGA/NHA stent has better biocompatibility.

4.3. Mechanical Properties of the Bracket. The ideal cartilage scaffold not only meets the strength similar to the specific part, but also needs to meet the compression mechanical properties similar to or the same as the specific part. Because the cartilage shaping process will be affected by the force of the cartilage, when the pressure on the cartilage tissue is reduced, the degree of cartilage absorption will be greater than the degree of cartilage reconstruction, and the cartilage tissue will be loose and fragile. If the compression force of the cartilage scaffold is different from that of the original laryngeal cartilage tissue after being implanted in a specific part, the pressure on the part with a small elastic modulus will be lower than the part with a large elastic modulus. The part creates a stress barrier to the part with low elastic modulus. If the elastic modulus of the cartilage scaffold is relatively small, the regeneration and shaping of the new cartilage tissue will be affected. However, if the elastic modulus of the cartilage scaffold is relatively large, it may cause osteoporosis in healthy cartilage tissue. The mechanical performance analysis results of the stent are shown in Figure 2. The mechanical properties of pure collagen scaffolds and mesenchymal stem cell composite porous scaffolds prepared by adding $0.1 \%, 0.3 \%$, and $0.5 \%$ PLGA, 
TABLE 2: Comparison of histological scores.

\begin{tabular}{lccc}
\hline Histological parameters & Control & PLGA-MSCs & PL GANHA-MSCs \\
\hline Smooth surface & $1.5(1-3)$ & $1.75(1-3)$ & $2(0-3)$ \\
Matrix & $1.125(0-3)$ & $1.375(0-3)$ & $2.125(1-4)$ \\
Newborn & $0.5(0-1)$ & $1.125(1-2)$ & $1.5(1-2)$ \\
Connect with surrounding cartilage & $1.457(0-2)$ & $1.6(0-2)$ & $1.725(1-2)$ \\
Chondrocyte distribution & $1.215(0-2)$ & $1.455(0-3)$ & $1.877(1-3)$ \\
Cell viability & $1.87(1-2)$ & $1.6(0-2)$ & $1.725(1-2)$ \\
\hline
\end{tabular}

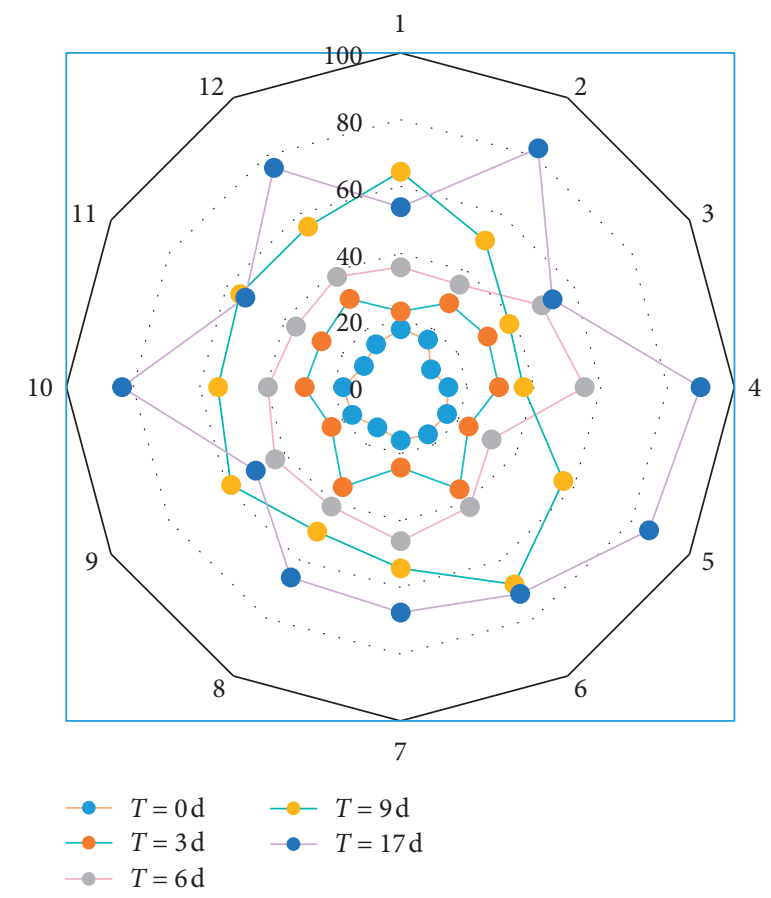

FIGURE 1: Growth and proliferation of cells in the scaffold.

respectively, under compression conditions are evaluated. It can be seen that as the content of PLGA increases, the compressive mechanical properties of the material gradually increase, and the modulus increases accordingly. Compared with the pure collagen-derived mesenchymal stem cell composite porous scaffold, the mechanical properties of the mesenchymal stem cell composite porous scaffold containing $0.5 \%$ PLGA have been greatly improved. The mechanical modulus increased from $39 \mathrm{kPa}$ to $95 \mathrm{kPa}$, and the mechanical strength increased from $0.46 \mathrm{MPa}$ to $1.1 \mathrm{MPa}$. It shows that, with the increase of PLGA concentration, the mechanical strength also increases positively. This shows that the residual carboxyl groups of PLGA are involved in the formation of collagen network and can react with the amino groups of collagen. This also realizes that PLGA is not physically added to the collagen scaffold. When the PLGA concentration is $0.5 \%$, the compressive strength reaches 1.1 $\mathrm{MPa}$, and the strength reaches a higher level, which can be used for cartilage repair. The increase of mechanical strength provides a good mechanical foundation and maneuverability for its medical and clinical development. Due to the addition of PLGA, the cross-linking reaction is more complete and the cross-linking density is also increased, which promotes the increase of mechanical properties. All these indicate that the addition of PLGA can effectively improve the mechanical properties of the collagen-mesenchymal stem cell composite porous scaffold.

4.4. Cytotoxicity Analysis. The results of the cytotoxicity analysis are shown in Figure 3. Cytotoxicity data shows that the growth of cells should increase with the increase of PLGA content, but when the concentration is $0.5 \%$, the growth of cartilage decreases slightly. It can be seen that the chondrocytes are scattered, not growing in pieces, and the number is small. On the hydrogel scaffolds containing $0.1 \%, 0.3 \%$, and $0.5 \%$ PLGA, the morphology of the cells is better, and the cells on some areas have grown into pieces. On the hydrogel scaffolds containing $0.3 \%$ and $0.5 \%$ PLGA, the number of cells is obviously increased and denser, and the cells can grow into pieces. This shows that the collagen hydrogel scaffold containing PLGA has good biocompatibility. The role of subchondral bone in cartilage regeneration is getting more and more attention. The subchondral bone not only provides support for the new cartilage layer, but also provides a suitable mechanical environment for the cartilage layer to promote cartilage regeneration. Due to the risk of stratification of the 


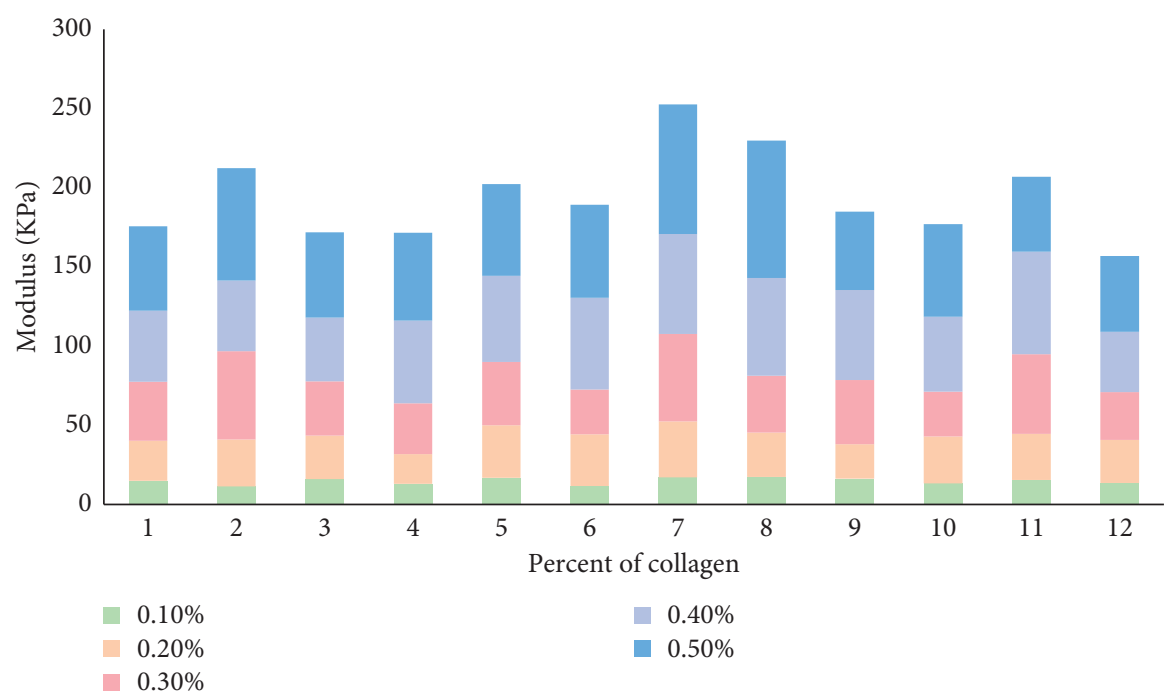

Figure 2: Analysis results of the mechanical properties of the stent.

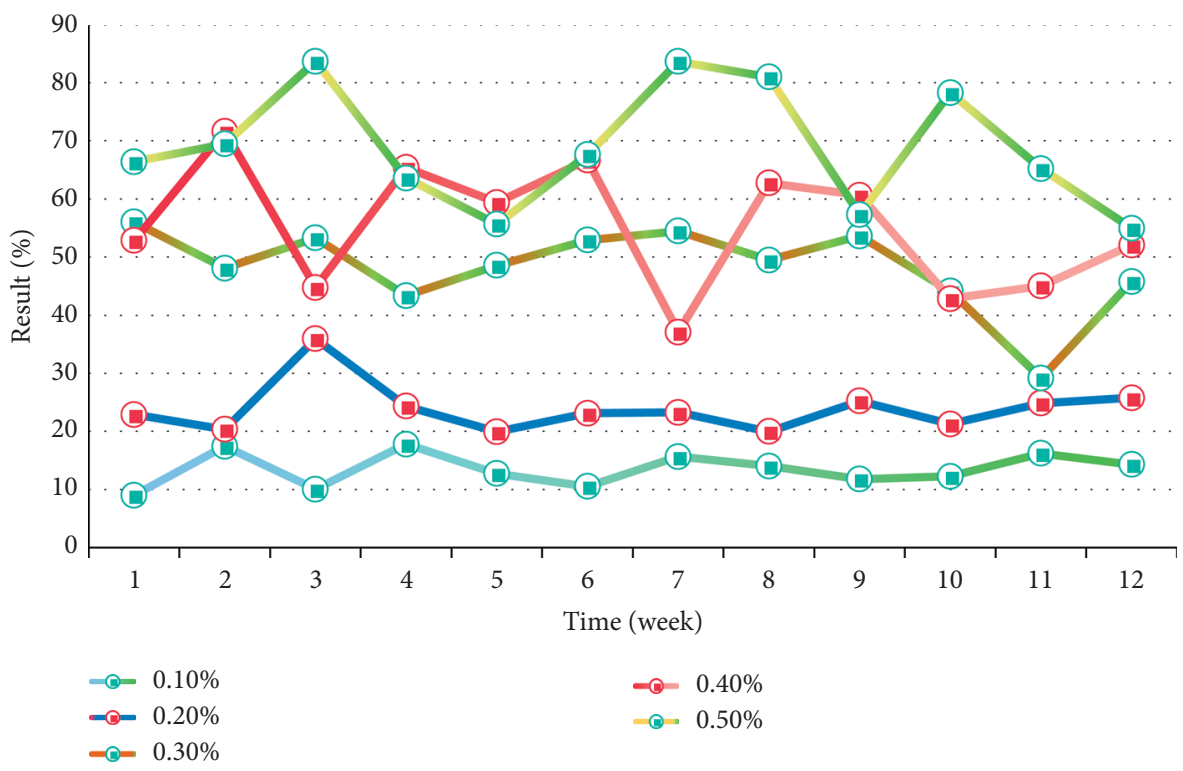

FIgURe 3: Cytotoxicity analysis results.

double-layer scaffold, in this study, we chose a single-layer scaffold to repair bone cartilage as found in the new bone tissue PLGA and PLGA better than NHA group and control group. This may be due to the fact that PLGA and NHA can provide a better biomechanical environment and the osteoinductive and osteoconductive activities of nanohydroxyapatite. The evaluation of biocompatibility and biodegradability in rats has confirmed that the scaffold promotes the formation of the scaffold. New blood vessels are formed around the implantation site. Within six weeks after implantation in the rat, the stent showed excellent absorption. The formation of new blood vessels observed in the newly formed connective tissue placed near the stent can completely restore blood flow.

All animals had no deaths, walking gait was normal, and the wounds had no bacterial infection. The knee joint has no deformity, redness, or swelling, and the range of motion is normal. The implant did not fall off during the operation. At 12 weeks postoperatively, cartilage-like tissue was seen in the repair area of the experimental group, with a small amount of connective tissue hyperplasia on the surface. The recovery of animals is shown in Figure 4. The surface of the joint is still uneven, with good continuity with the surrounding cartilage, and there is still a depression. Defect repair: at 6 weeks, the cartilage defect repaired in the PLGA group was rough and slightly yellow, and the boundary was clear; the cartilage defect repaired in the PLGA/NHA group was flush with the surrounding cartilage tissue, and the boundary was blurred; the cross section showed only a new layer of the defect filmlike structure formed. At 12 weeks, the repaired tissue surface of the defect in the PLGA group was irregular, still concave, with poor integration, regenerated cartilage was slightly white, and the boundary between repaired tissue and 


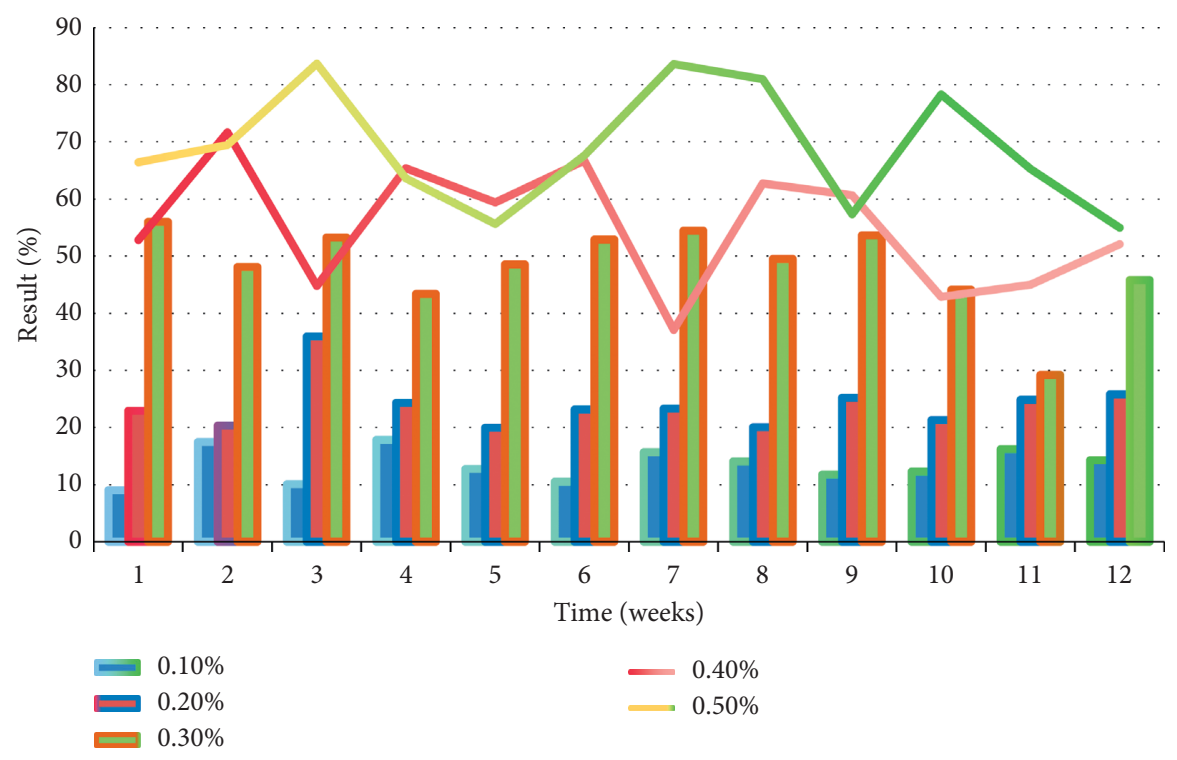

FIgURe 4: Animal recovery.

autologous tissue was clear. The color and texture of the new cartilage in the PLGA/NHA group were similar to the surrounding normal cartilage, and the boundary was blurred. The remaining stent components can only be seen in the longitudinal section. In the blank group, fibrous tissue was clearly visible, and the surface was not smooth. No heterotopic ossification was found in each specimen.

\section{Conclusion}

Polymer biomaterials have an important influence in medical technology. Polymer hydrogel through an experimental design is used in the first human biological material. This article outlines the relevant characteristics of synthetic and natural polymer-derived hydrogels and their use in tissue engineering. It discusses examples of responsive hydrogels used to design methods using multidisciplinary methods. The manufacturing examples of polymer-based biomaterials are also described in detail. Since highly porous three-dimensional scaffolds are essential in cellular processes, for tissue engineering, the latest developments in effective methods for scaffold manufacturing are described. In addition, this study also clarified the incorporation of factor molecules for enhancing tissue formation and its controlled release. Finally, the future challenges of effectively manufacturing effective polymer biomaterials for tissue regeneration and medical device applications are discussed.

In this study, rat bone marrow mesenchymal stem cells were obtained by the whole bone marrow washing method, and the cells were inoculated into cell culture flasks. When the primary cultured cells proliferated, trypsin digestion was used to open the cell link, and then the serum medium was added to terminate the cells digestion, passage, and expansion according to cell density. PLGA/NHA and polylactic acid-glycolic acid (PLGA) are heated under ultrasonic vibration until a uniform PLGA/NHA and PLGA solution is obtained. Then, the sample was added to the tubular mold, heated, and cooled to obtain the mesenchymal stem cell composite porous scaffold. The MSCs cell suspension is drawn into the needle from the inside of the stent with a microinjector each time, and the cell suspension is dropped on the outside of the stent to ensure that there are composite cells inside and outside the stent. The skin subcutaneous tissue is cut along the inner side of the rat's knee joint and the nodular sac is cut. The stent materials are filled into the osteochondral defect to observe the cartilage healing of the rat.

Cartilage-like tissues were seen in the repair area of the experimental group after surgery, with a small amount of connective tissue hyperplasia on the surface. The surface of the joint is still uneven, with good continuity with the surrounding cartilage, and there is still a depression. Defect repair: at the beginning, the cartilage defect repaired in the PLGA group was rough and slightly yellow, and the boundary was clear; the cartilage defect repaired in the PLGA/NHA group was flush with the surrounding cartilage tissue, and the boundary was blurred; the cross section showed that only one layer was newly formed at the defect, the film-like structure. In the PLGA group, the defect of the repaired tissue surface was irregular, there were still depressions and poor integration, regenerated cartilage was slightly white, and the boundary between repaired tissue and autologous tissue was clear. The color and texture of the new cartilage in the PLGA/NHA group were similar to the surrounding normal cartilage, and the boundary was blurred. The remaining stent components can only be seen in the longitudinal section. In the blank group, fibrous tissue was clearly visible, and the surface was not smooth. No heterotopic ossification was found in each specimen. The mesenchymal stem cell composite porous scaffold designed in this study has good compatibility and is beneficial to repairing sports cartilage injury. 


\section{Data Availability}

The data that support the findings of this study are available from the corresponding author upon reasonable request.

\section{Conflicts of Interest}

The author declares that there are no conflicts of interest with respect to the research, authorship, and/or publication of this article.

\section{References}

[1] L. C. Yong, N. F. Abdul Malek, E. N. Se Yong, W. H. Yap, M. Nobuyuki, and N. Yoshitaka, "Fabrication of hydroxyapatite blended cyclic type polylactic acid and poly ( $\varepsilon$-caprolactone) tissue engineering scaffold," International Journal of Applied Ceramic Technology, vol. 16, no. 2, pp. 455-461, 2019.

[2] E. A. Masoudi, J. Ribas, G. Kaushik, J. Leijten, and A. Khademhosseini, "Platelet-rich blood derivatives for stem cell-based tissue engineering and regeneration," Current Stem Cell Reports, vol. 2, no. 1, pp. 33-42, 2016.

[3] E. A. Naumenko, I. D. Guryanov, R. Yendluri, Y. M. Lvov, and R. F. Fakhrullin, "Clay nanotube-biopolymer composite scaffolds for tissue engineering," Nanoscale, vol. 8, no. 13, pp. 7257-7271, 2016.

[4] M. Emmert, P. Witzel, and D. Heinrich, "Challenges in tissue engineering - towards cell control inside artificial scaffolds," Soft Matter, vol. 12, no. 19, pp. 4287-4294, 2016.

[5] C. Tangsadthakun, S. Kanokpanont, and N. Sanchavanakit, "Properties of collagen/chitosan scaffolds for skin tissue engineering," Journal of Metals, vol. 16, no. 16, pp. 37-44, 2017.

[6] A. M. Boos, A. Weigand, R. Brodbeck, J. P. Beier, A. Arkudas, and R. E. Horch, "The potential role of telocytes in tissue engineering and regenerative medicine," Seminars in Cell \& Developmental Biology, vol. 55, no. 6, pp. 70-78, 2016.

[7] P. Andrea, L. Luigi, and G. Marco, "Decellularized hydrogels in bone tissue engineering: a topical review," International Journal of Medical Ences, vol. 15, no. 5, pp. 492-497, 2018.

[8] T. Trzeciak, M. Richter, W. Suchorska et al., "Application of cell and biomaterial-based tissue engineering methods in the treatment of cartilage, menisci and ligament injuries," International Orthopaedics, vol. 40, no. 3, pp. 615-624, 2016.

[9] F. M. Chen, "Periodontal tissue engineering and regeneration," Chinese Journal of Stomatology, vol. 52, no. 10, pp. 610-614, 2017.

[10] A. K. Nguyen and R. J. Narayan, "Liquid-phase laser induced forward transfer for complex organic inks and tissue engineering," Annals of Biomedical Engineering, vol. 45, no. 1, pp. 84-99, 2017.

[11] J. Gilmore, T. Burg, R. E. Groff, and K. J. L. Burg, "Design and optimization of a novel bio-loom to weave melt-spun absorbable polymers for bone tissue engineering," Journal of Biomedical Materials Research Part B: Applied Biomaterials, vol. 105, no. 6, pp. 1342-1351, 2017.

[12] B. Pei, W. Wang, Y. Fan, X. Wang, F. Watari, and X. Li, "Fiber-reinforced scaffolds in soft tissue engineering," Regenerative Biomaterials, vol. 4, no. 4, pp. 257-268, 2017.

[13] S. Kazemirad, H. K. Heris, and L. Mongeau, "Viscoelasticity of hyaluronic acid-gelatin hydrogels for vocal fold tissue engineering," Journal of Biomedical Materials Research Part B: Applied Biomaterials, vol. 104, no. 2, pp. 283-290, 2016.
[14] M. F. Griffin, Y. Premakumar, A. M. Seifalian, M. Szarko, and P. E. M. Butler, "Biomechanical characterisation of the human auricular cartilages; implications for tissue engineering," Annals of Biomedical Engineering, vol. 44, no. 12, pp. 34603467, 2016.

[15] R. Trombetta, J. A. Inzana, E. M. Schwarz, S. L Kates, and H. A Awad, "3D printing of calcium phosphate ceramics for bone tissue engineering and drug delivery," Annals of Biomedical Engineering, vol. 45, no. 1, pp. 23-44, 2017.

[16] G. Gao and X. Cui, "Three-dimensional bioprinting in tissue engineering and regenerative medicine," Biotechnology Letters, vol. 38, no. 2, pp. 203-211, 2016.

[17] M. C. M. Conde, L. A. Chisini, F. F. Demarco, J. E. Nör, L. Casagrande, and S. B. C. Tarquinio, "Stem cell-based pulp tissue engineering: variables enrolled in translation from the bench to the bedside, a systematic review of literature," International Endodontic Journal, vol. 49, no. 6, pp. 543-550, 2016.

[18] J. Fan and J. Yang, "Preparation and characterization of a chitosan/galactosylated hyaluronic acid/heparin scaffold for hepatic tissue engineering," Journal of Biomaterials Science Polymer Edition, vol. 28, no. 6, pp. 1-33, 2017.

[19] S. Stratton, N. B. Shelke, K. Hoshino, S. Rudraiah, and S. G. Kumbar, "Bioactive polymeric scaffolds for tissue engineering," Bioactive Materials, vol. 1, no. 2, pp. 93-108, 2016.

[20] M. W. Laschke and M. D. Menger, "Prevascularization in tissue engineering: current concepts and future directions," Biotechnology Advances, vol. 34, no. 2, pp. 112-121, 2016.

[21] F. Menaa, A. Abdelghani, and B. Menaa, "Graphene nanomaterials as biocompatible and conductive scaffolds for stem cells: impact for tissue engineering and regenerative medicine," Journal of Tissue Engineering and Regenerative Medicine, vol. 9, no. 12, pp. 1321-1338, 2016.

[22] F. Khan, M. Tanaka, and S. R. Ahmad, "Fabrication of polymeric biomaterials: a strategy for tissue engineering and medical devices," Journal of Materials Chemistry B, vol. 3, no. 42, pp. 8224-8249, 2016.

[23] K. Schütz, A.-M. Placht, B. Paul, S. Brüggemeier, M. Gelinsky, and A. Lode, "Three-dimensional plotting of a cell-laden alginate/methylcellulose blend: towards biofabrication of tissue engineering constructs with clinically relevant dimensions," Journal of Tissue Engineering and Regenerative Medicine, vol. 11, no. 5, pp. 1574-1587, 2017.

[24] A. Pahnke, G. Conant, L. D. Huyer, Y. Zhao, N. Feric, and M. Radisic, "The role of WNT regulation in heart development, cardiac repair and disease: a tissue engineering perspective," Biochemical and Biophysical Research Communications, vol. 473, no. 3, pp. 698-703, 2016.

[25] P. Wang, T. Yao, Z. Li et al., "A superhydrophobic/electrothermal synergistically anti-icing strategy based on graphene composite," Composites Science and Technology, vol. 198, Article ID 108307, 2020.

[26] O. Akhavan, "Graphene scaffolds in progressive nanotechnology/stem cell-based tissue engineering of the nervous system," Journal of Materials Chemistry B, vol. 4, no. 19, pp. 3169-3190, 2016. 